\title{
Thyroid storm: A rare and life-threatening cause of acute neurological manifestations during urgent percutaneous coronary intervention for acute coronary syndrome
}

\author{
Sophie Degrauwe ${ }^{1}$, Laura Marino ${ }^{2}$, Jihen Ayari ${ }^{1}$, Andrea Zuffi ${ }^{1}$, \\ Olivier Muller ${ }^{1}$, Eric Eeckhout ${ }^{1}$, Juan F. Iglesias ${ }^{1}$ \\ ${ }^{1}$ Department of Cardiology, Lausanne University Hospital, Lausanne, Switzerland \\ ${ }^{2}$ Department of Endocrinology, Lausanne University Hospital, Lausanne, Switzerland
}

Acute neurological complications during coronary angiography (CAG) are extremely uncommon with an incidence of $<0.1 \%$ [1] but they remain a challenging clinical problem, particularly in patients with acute coronary syndrome. Periprocedural ischemic or hemorrhagic stroke remains the most common cause with an incidence of $0.07-0.38 \%$ and is associated with high rates of morbidity and mortality [2]. However, sudden-onset neurological symptoms during diagnostic CAG or percutaneous coronary intervention (PCI) may result from rarer albeit fatal neurological or endocrine conditions, such as seizure, encephalopathy, contrast media-induced neurotoxicity or acute thyrotoxicosis [3]. Thyroid storm (TS) is a life-threatening endocrine emergency that represents the extreme manifestation of acute thyrotoxicosis and is associated with high morbidity and mortality $(10 \%)$ rates if left untreated $[3,4]$.

Herein is reported a 70-year-old woman with arterial hypertension, pacemaker implantation for sick sinus syndrome and paroxysmal atrial fibrillation on long-term amiodarone therapy, who was admitted to the emergency department with acute typical chest pain. The patient underwent previous coronary artery bypass grafting with left internal mammary artery (LIMA) to the left anterior descending artery (LAD) and saphenous vein graft to the right coronary artery (RCA) 16 years earlier, followed by PCI to the distal RCA with a drugeluting stent 9 years later. Three weeks prior to admission, the patient presented with weight loss, tremor and palpitations suggestive of thyrotoxicosis, with decreased levels of thyroid stimulating hormone $(<0.005 \mathrm{mIU} / \mathrm{L}$, normal range $0.4-$ $-4.0 \mathrm{mIU} / \mathrm{L}$ ), and elevated levels of free thyroxine (T4) $(93 \mathrm{pmol} / \mathrm{L}$, normal range $11-27 \mathrm{pmol} / \mathrm{L})$ and free tri-iodothyronine (T3) $(16.5 \mathrm{pmol} / \mathrm{L}$, normal range 0.8-2.7 pmol/L), suggestive of amiodarone-induced thyrotoxicosis (anti-TSH receptor antibody: $<3 \mathrm{U} / \mathrm{L}$ ). Amiodarone therapy was discontinued and an antithyroid drug (carbimazole $20 \mathrm{mg}$ /day) was initiated with reduction of free $\mathrm{T} 4(70 \mathrm{pmol} / \mathrm{L})$ and T3 (15 pmol/L) levels at 3-week follow-up. The thyroid ultrasound showed a slightly enlarged and heterogeneous thyroid gland, but excluded a nodular formation as well as hypervascularisation. Upon present admission, electrocardiogram demonstrated ST-segment depression from V3 to V6 leads and cardiac biomarkers were elevated. The CAG demonstrated a 3-vessel coronary artery disease with occlusion of the saphenous vein graft to RCA, involution of the LIMA graft to LAD, $50 \%$ distal left main coronary artery stenosis, obtuse marginal branches and intermediate artery stenoses, $50 \%$ distal RCA in-stent restenosis, and hemodynamically significant proximal LAD stenosis. The patient underwent PCI to the proximal LAD with a drug-eluting stent. During the procedure, the patient developed acute neurological symptoms including headache, agitation, delirium, confusion, nausea and vomiting, that required

Address for correspondence: Juan F. Iglesias, MD, Department of Cardiology, Lausanne University Hospital (CHUV),

Rue du Bugnon 46, 1011 Lausanne, Switzerland, tel: +41 2131400 10, fax: +4121 31400 13,

e-mail: Juan-Fernando.Iglesias@chuv.ch

Received: 02.11.2016 Accepted: 26.01.2017 


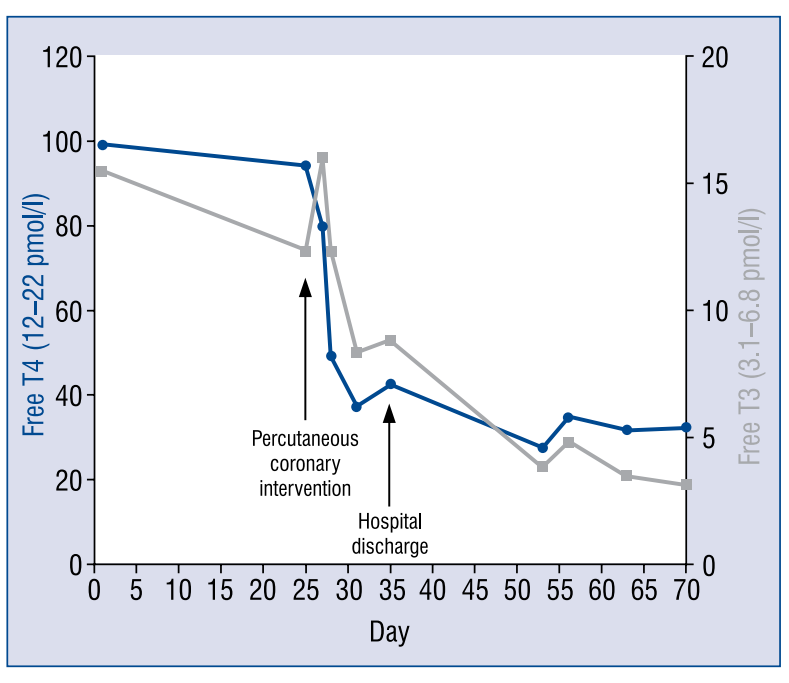

Figure 1. Temporal trends in circulating levels of free thyroid hormones.

premature interruption of the procedure. Urgent physical examination showed no focal neurological symptoms, but revealed fever $\left(38.5^{\circ}\right)$ and sinus tachycardia (110 bpm). An urgent cerebral computed tomography scan excluded acute intracranial hemorrhage or ischemic stroke. Electroencephalogram showed moderate and diffuse encephalopathy without irritative pattern. Acute thyrotoxicosis with TS precipitated by iodinated contrast media administration during PCI was suspected. Clinical presentation was meeting the diagnostic criteria of the Japan Thyroid Association and the Burch and Wartofsky's score was 55 points. Immediate therapy was initiated including intravenous hydrocortisone, metoprolol and increase of carbimazole dosage. The clinical course was favourable with total regression of neurological manifestations on the following day and progressive decrease of free T4 and T3 levels (Fig. 1).

Acute TS is an extremely rare hypermetabolic condition induced by excessive thyroid hormones release in individuals with thyrotoxicosis with an annual incidence of $2 / 1,000,000$ patients, but it remains associated with high mortality rates $(10-30 \%)[3,4]$. TS is a clinical diagnosis considering the absence of absolute circulating thyroid hormones levels cut-off and usually occurs following a precipitating event in patients with severe thyrotoxicosis or previously undiagnosed hyperthyroidism [3]. TS results from sudden inhibition of thyroid hormone binding to plasma proteins leading to further increase in thyroid hormone levels. The main precipitating factors include irregular use or discontinuation of antithyroid drugs as well as iodinated contrast media administration. However, no clear trigger may be identified in $25-43 \%$ of patients [3]. The Burch and Wartofsky's score is a scoring system based on clinical features proposed to guide the diagnosis of TS, particularly when thyroid hormone assays are not readily available [5]. Considering high mortality rates [4], medical treatment of TS should be initiated promptly and be aimed at treating the underlying condition, alleviating catecholamine-dependent mechanisms and peripheral effects of thyroid hormone excess, inhibiting new thyroid hormone synthesis and release, removing circulating thyroid hormones and enhancing thyroid hormone clearance [3, 6]. Acute thyrotoxicosis with abrupt onset of neurological symptoms and delirium precipitated by administration of iodinated contrast media during CAG procedures has been previously reported [3]. Here, described for the first time as research indicates, is the unique case of a patient with recent-onset hyperthyroidism who developed acute delirium and confusional state caused by acute TS during emergent PCI for acute coronary syndrome. Our case highlights the critical importance of the prompt recognition and aggressive management of acute TS to prevent high risk of morbidity and mortality that may be associated with this alternative, life-threatening and potentially increasing cause of acute neurological manifestations in patients with hyperthyroidism undergoing PCI using iodinated contrast media.

\section{Conflict of interest: None declared}

\section{References}

1. Duffis EJ, Jones D, Tighe D, et al. Neurological complications of coronary angiographic procedures. Expert Rev Cardiovasc Ther. 2007; 5(6): 1113-1121, doi: 10.1586/14779072.5.6.1113, indexed in Pubmed: 18035927.

2. Khatri P, Kasner SE. Ischemic strokes after cardiac catheterization: opportune thrombolysis candidates? Arch Neurol. 2006; 63(6): 817-821, doi:10.1001/archneur.63.6.817, indexed in Pubmed: 16769862 .

3. Chiha M, Samarasinghe S, Kabaker AS. Thyroid storm: an updated review. J Intensive Care Med. 2015; 30(3): 131-140, doi:10.1177/0885066613498053, indexed in Pubmed: 23920160.

4. Akamizu T, Satoh T, Isozaki O, et al. Japan Thyroid Association. Diagnostic criteria, clinical features, and incidence of thyroid storm based on nationwide surveys. Thyroid. 2012; 22(7): 661-679, doi: 10.1089/thy.2011.0334, indexed in Pubmed: 22690898.

5. Burch HB, Wartofsky L. Life-threatening thyrotoxicosis. Thyroid storm. Endocrinol Metab Clin North Am. 1993; 22(2): 263-277, indexed in Pubmed:8325286.

6. Satoh T, Isozaki O, Suzuki A, et al. 2016 Guidelines for the management of thyroid storm from The Japan Thyroid Association and Japan Endocrine Society (First edition). Endocr J. 2016; 63(12): 1025-1064, doi: 10.1507/endocrj.EJ16-0336, indexed in Pubmed: 27746415. 